\title{
Abbreviations
}

The following are abbreviations used throughout the book.

\section{States of the United States}

AL Alabama

AK Alaska

AR Arkansas

AZ Arizona

CA California

CO Colorado

CT Connecticut

DE Delaware

DC District of Columbia

FL Florida

GA Georgia

HI Hawaii

IA Iowa

ID Idaho

IL Illinois

IN Indiana

KS Kansas

KY Kentucky

LA Louisiana

MA Massachusetts

ME Maine

MD Maryland

MI Michigan

MN Minnesota

MO Missouri

MS Mississippi
MT Montana

NC North Carolina

ND North Dakota

NE Nebraska

NH New Hampshire

NJ New Jersey

NM New Mexico

NV Nevada

NY New York

$\mathrm{OH}$ Ohio

OK Oklahoma

OR Oregon

PA Pennsylvania

PR Puerto Rico

RI Rhode Island

SC South Carolina

SD South Dakota

TN Tennessee

TX Texas

UT Utah

VA Virginia

VT Vermont

WA Washington

WI Wisconsin

WV West Virginia

WY Wyoming

\section{Canadian Provinces}

Alta. Alberta

B.C. British Columbia

Lab. Labrador

Man. Manitoba

M.P. Maritime Provinces

N.B. New Brunswick

Nfdl. Newfoundland

N.S. Nova Scotia

N.W.T. Northwest Territories

Ont. Ontario

P.E.I. Prince Edward Island

Que. Quebec

Sask. Saskatchewan

Y.T. Yukon Territory 

Parasites of

North American Freshwater Fishes 
